\title{
Analysis of the Construction of Online Open Courses under the Background of Internet + Education-Take Henan Province as an example
}

\author{
Bo Zeng ${ }^{1,2, a}$, Shanshan $\mathrm{Li}^{2, b,{ }^{*}}$ and Zhixue Zhang ${ }^{2, \mathrm{c}}$ \\ 1 School of Information Technology, Luoyang Normal University, China \\ ${ }^{2}$ Network Information Center, Henan University of Science and Technology, China \\ awbzeng_hn@163.com, blss@haust.edu.cn, 'zhangzx@haust.edu.cn \\ * Shanshan Li
}

Keywords: Internet+Education, Henan, online open courses, resources.

\begin{abstract}
As the main means of realization of "Internet + Education", the online open course has fully utilized the open and sharing characteristics of the Internet, established a new educational concept, reformed the educational model, and brought a new industry form for education. The paper analyzes in detail the problems existing in the process of online open course construction in Henan Province, and puts forward suggestions and countermeasures from the aspects of curriculum resource construction, promotion and utilization.
\end{abstract}

\section{互联网+背景下在线开放课程建设情况分析}

\author{
曾波 ${ }^{1,2, a}$, 李姗姗 $2, b,{ }^{*}$, 张治学 $2, c$ \\ 1洛阳师范学院信息技术系, 洛阳, 河南, 中国 \\ 2河南科技大学网络信息中心，洛阳，河南，中国 \\ awbzeng_hn@163.com, blss@haust.edu.cn, c zhangzx@haust.edu.cn \\ “李姗姗
}

关键词：互联网+; 河南; 在线开放课程; 资源

中文摘要：在线开放课程作为 “互联网+教育” 的主要实现手段充分利用了互联网的开 放、共享特点, 建立了新的教育理念, 改革了教育模式, 为教育事业带来了新的行业形 态。论文详细分析了河南省在线开放课程建设过程中存在的问题, 并从课程资源建设、推 广、利用等方面提出了建议与对策。

\section{1. 引言}

2015年李克强总理在政府工作报告中提出了“互联网+”的概念，并制定了11项互联网+重 点行动计划, 自此“互联网+”开始渗入各行各业, 影响和重塑着各行各业的结构, 在教育领域, “互联网+”正在深刻影响及变革着教育理念和教育体制。“互联网+”指的是把互联网的创新成 果与经济社会各领域深度融合，推动技术进步、效率提升和组织变革，提升实体经济创新力 和生产力, 形成更广泛的以互联网为基础设施和创新要素的经济社会发展新形态。互联网以 开放、协作、分享，平等，自由为其主要特点，“互联网＋教育”一方面将先进的信息技术引入 教育领域, 实现教学资源数字化, 另一方面, 将互联网的核心特点“开放、共享”引入了教育领 域, 实现了数字化教学资源面向学习者的开放, 面向教师的共享。互联网与教育领域的深度 
融合为传统教育事业带来了新的行业形态, 并极大地改变了教育模式与教育理念, 掀起了教 育改革的序曲。当前, 互联网十教育已经成为教育界的热门话题, 围绕该话题的各种形式的 教学实践探索与学术研究正广泛而深入地进行。互联网与教育的深度融合, 将传统的教育和 学习方式发生根本改变, 也必然给在线教育带来全新的挑战和千载难逢的发展机遇。在线开 放课程作为“互联网+教育”的发展前沿, 也必然会蓬勃发展, 将教育改革进一步深化。

\section{2. 现状}

在线开放课程已经成为当前高等教育改革的主流方式之一, 必然成为未来教育的发展趋 势。本节主要描述在线开放课程概念的起源、演变，发展阶段。

在线开放课程起源于2001年美国麻省理工学院 (Massachusetts Institute of Technology, MIT) 的“开放课件”（Open Course Ware，OCW）项目 [1]，MIT计划用10年时间通过该项目将其所有 的课程资源公开发布到互联网, 供全球网络用户免费使用。OCW项目强调的是资源的共享, 并不介入利用这些资源学习的学习者的具体学习过程, 也不提供学习帮助和承认学习的有效 性。尽管如此, OCW项目成功打破了名校资源封闭使用的局面, 开启了世界开放教育运动的 大门，在高等教育的发展历史上具有里程碑的意义。

2002年，联合国教科文组织提出了开放教育资源（Open Educational Resource，OER）的 概念 ${ }^{[2]}$ 。OER扩展了 OCW 的概念范围, 其主要包括了三个方面的内容: 学习工具、学习内容 和实施资源。OER强调了教育资源的重用和共享, 对高等教育的教学、学习和教学研究产生 了巨大影响。

2008年，在上述概念的铺垫下，第一门具有开放学习意义的网上课程“联通主义和连接性 知识”在马尼托巴大学由乔治. 西蒙斯和斯蒂芬. 唐斯开设, 值得注意的是, 参与在线课程学习 的学习者包括两类：（1）在校注册的25名学生; (2) 来自于世界各地的2300名校外未注册 学生。所有学生均需要借助于各类网络学习工具, 如博客, RSS订阅等完成课程学习活动。这 门课程具有了大规模（Massive）、开放性（Open）、在线学习（Online）的特点。后来, 戴 维.科米尔 (Dave Cormier) 使用了MOOC来描述该课程, 因而该课程被认为是一门真正意义 上的MOOC课程。

2012年MOOC以风暴之势席卷了全球，被称为“MOOC元年”。2013年，美国新媒体联盟发 布了《地平线报告》（高教版）指出: 未来1 5年之内, MOOC、在线学习、非正式学习、翻 转课堂、学习分析等将成为高校教学的主流技术 ${ }^{[3]}$ 。

考虑到MOOC分类的多样性，在以支撑的基础学习理论为标准下，MOOC可被划分为

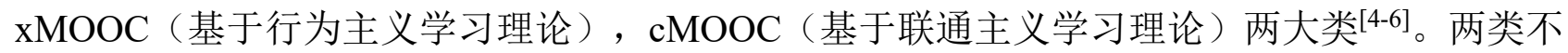
同的MOOC又各自发展出若干开放学习支持平台, 其中 $\mathrm{xMOOC}$ 的主流支持平台为 $\mathrm{Edx}$ 、 Udacity, Coursera; 而cMOOC的代表性平台为Udemy。当前国外MOOC主流平台统计如表1所 示。

表1 国外主流MOOC平台

\begin{tabular}{|c|c|c|c|c|c|}
\hline 平台名称 & 创建者 & 盈利性质 & 支持的移动终端 & 建设时间 & 合作院校 \\
\hline Edx & MIT 和哈佛 & 非盈利 & 无 & 2012 & 全世界顶尖的大学 \\
\hline Udacity & 斯坦福大学 & 盈利 & Ios 系统 & 2011 & $\begin{array}{c}\text { 主要计算机领域的科技公 } \\
\text { 司如: Googl 、微软等 }\end{array}$ \\
\hline Coursera & 斯坦福大学 & 盈利 & Android 和 ios 系统 & 2012 & 全世界顶尖的大学和机构 \\
\hline
\end{tabular}


国内MOOC主流平台统计 ${ }^{[7]}$ 如表2所示。

表2：国内主流MOOC平台

\begin{tabular}{|c|c|c|c|c|c|}
\hline 平台名称 & 创建者 & 盈利性质 & 课程来源 & 建设时间 & 开放范围 \\
\hline 学堂在线 & 清华大学 & 盈利 & 清华大学 & 2014 & 面向公众 \\
\hline 好大学在线 & 上海交通大学 & 公益 & $\begin{array}{c}\text { 上海交通大学 } \\
\text { 及各高校 }\end{array}$ & 2014 & 面向在校学生及公众 \\
\hline $\begin{array}{c}\text { 中国大学 } \\
\text { MOOC }\end{array}$ & 网易和爱课程 & 盈利 & 高校联盟 & 2014 & $\begin{array}{c}\text { 学校专有课程面向在校学 } \\
\text { 生、慕课面向公众 }\end{array}$ \\
\hline 智慧树 & $\begin{array}{c}\text { 卓越䜭新数码 } \\
\text { 公司与东西部 } \\
\text { 高校课程共享 } \\
\text { 联盟 }\end{array}$ & 盈利 & 社会团体 & 2014 & 面向在校学生及公众 \\
\hline 超星慕课 & 超星集团 & 盈利 & 个体学者 & 2014 & 面向公众 \\
\hline
\end{tabular}

\section{3. 河南省在线开放课程建设现状及问题分析}

2015年，根据教育部《关于加强高等学校在线开放课程建设应用与管理的意见》（教高 （2015）3号）、河南省人民政府《关于深化高等教育综合改革全面提升服务经济社会发展能 力的意见》（豫政〔2015〕41号）、河南省人民政府《关于加快推进教育信息化建设工作的意 见》（豫政(2015) 16号) 要求, 河南省正式迈开了精品在线开放课程建设的步伐。三年来, 河南省高校共建设省级在线开放课程284门，其中2016年建设87门，2017年建设95门，2018年 建设102门。总体来说, 课程建设每年呈现增长的趋势。考虑到2018年新立项的大部分在线开 放课程并没有上线运行，本文主要以2016年和2017年的在线开放课程数据为基础进行分析。

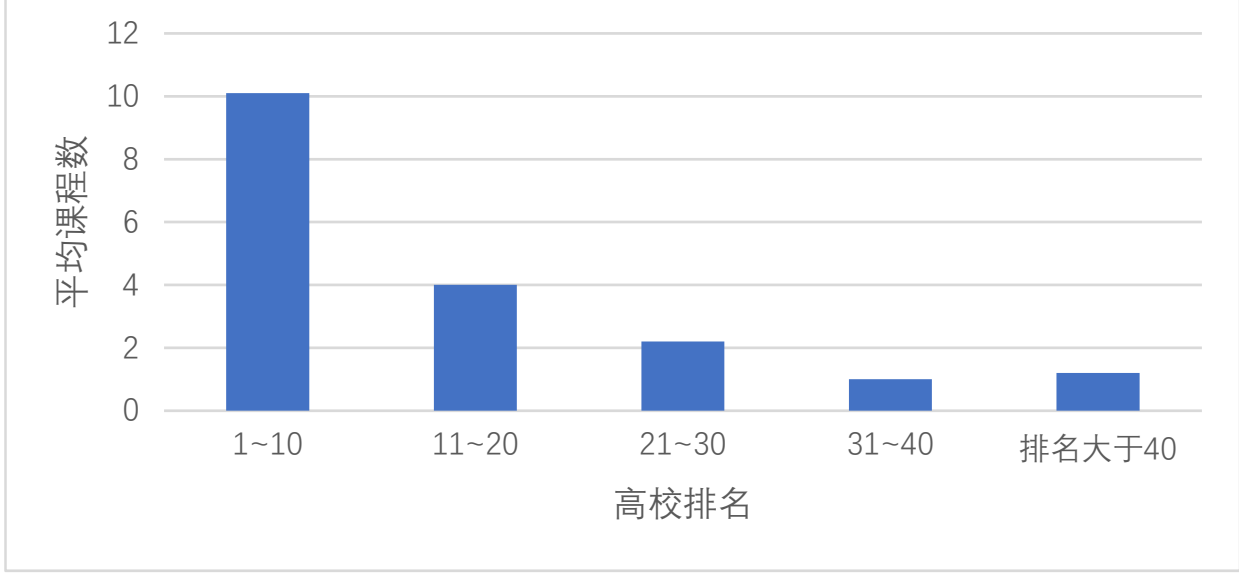

图1 河南省在线开放课程分布情况

图1给出的是河南省2016-2017年建设的省级在线开放课程的分布情况。河南省规划建设 共计 300门省级在线开放课程, 涉及131所高校（包括高职高专院校）。图中所用到的高校排 名数据为 2018 年校友会公开的河南省高校排名数据。从图中可以看出, 河南省排名前十的高 校所建设的在线课程门数平均在 $10 门$ 左右。事实上, 在前十高校中, 入选双一流高校的郑州 大学与河南大学的课程数共计为 $40 门$, 占比约 $40 \%$ 。而在排名为 $11 \sim 20$ 的十所高校中, 平均在 线课程门数仅有 $4 门$, 不到前十高校平均课程数的 $50 \%$ 。值得注意的是排名超过 20 名的高校, 平均建课数为不超过 $2 门$ 门这表明在剩余的 121 所高校中, 绝大部分高校的在线课程门数仅为 1门。 


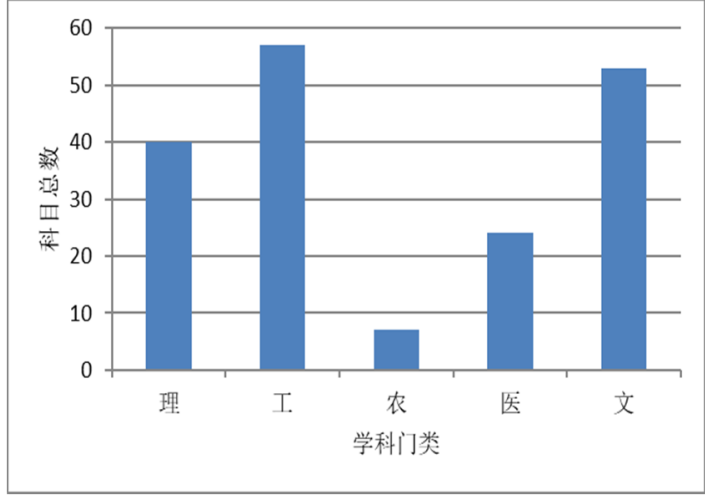

图2 河南省在线开放课程学科门类统计

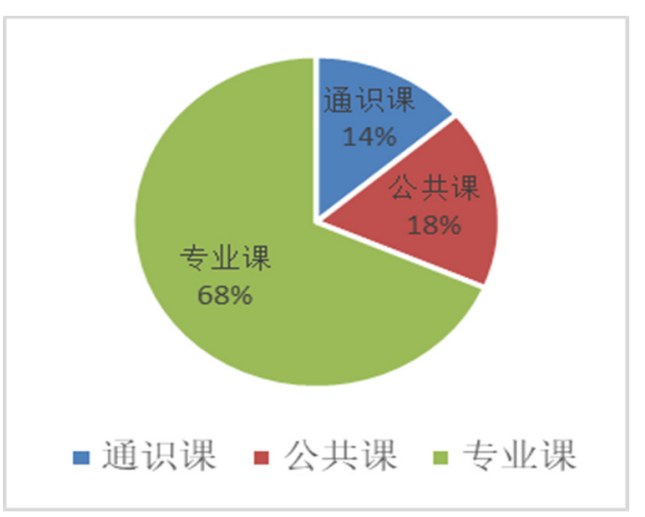

图3 河南省在线开放课程性质分布

图2给出的是河南省在线开放课程学科门类统计情况。该图显示在目前已建设的181门在 线开放课程中, 理科课程门数为 40 门, 工科课程门数为 $57 门$ 门农学类课程门数为 7 门, 医学类 课程门数为 $24 门$, 文科课程门数为 53 门。理、工、文三个科目的课程建设情况比较理想, 特 别是工科和文学基本持平, 但农学和医学类的课程建设数据偏低, 特别是农学类的课程, 仅 占总开设课程的 $2.4 \%$ 。

图3给出的是河南省在线开放课程的性质统计, 从统计结果来看, 在所建设的在线开放课 程中, 通识课程 $25 门$ 门, 公共课程 32 门, 专业课程 124 门。专业课程占所有建设课程的 $68 \%$, 这 表明河南省高校在线开放课程建设资源主要集中在专业课类别。各高等学校立足于自身学科 特色, 积极建设能够显示自身学科或专业优势的专业课程, 希望通过MOOC来扩大自身的影 响力与名声, 从而吸引相关资源, 聚合在一起实现优势共享。

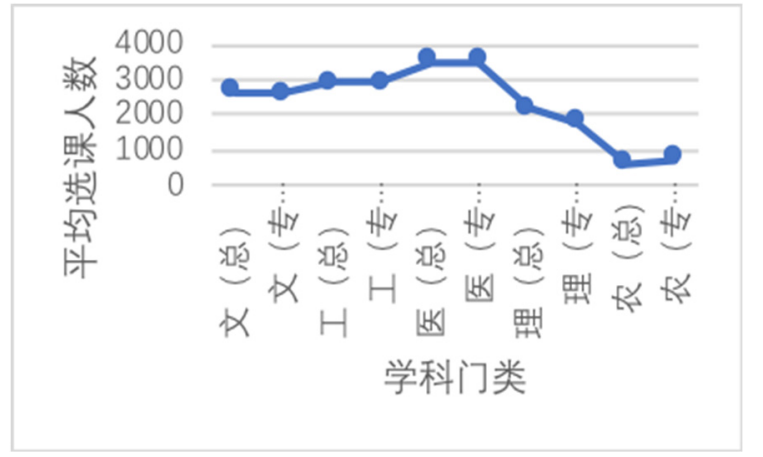

图4 各学科平均选课人数分布

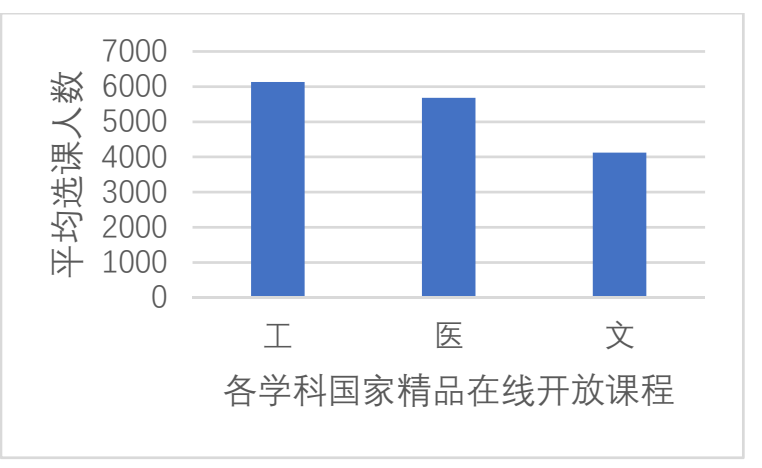

图5 国家精品在线开放课程平均选课人数

图4给出的是 2016 年河南省上线的在线开放课程中, 各学科的平均选课人数。每门学科包 含了两类数据, 一类是含有公共课, 通识课的平均选课人数, 一类是仅包含专业课的平均选 课人数。从图中可以看出: 文、工, 医学类课程的平均选课人数较高, 平均超过 2500 人, 其 中医学类课程平均选择人数达到 3000 人左右, 理学类课程选课人数略低于前述 $3 门$ 学科, 但平 均选课人数超过了 1500 ; 相比其它学科而言, 农学类课程平均选课人数最低, 不足 1000 人。 与目前主流MOOC平台课程相比, 选课人数偏低 ${ }^{[8]}$, 其主要原因在于课程影响范围较窄, 限制 了学习者类型与人数。

图5给出的是国家精品在线开放课程的平均选课人数分布（注意：由于理科类没有课程入 选国家精品在线开放课程, 故统计中缺乏相关数据项）。图中数据表明：相比非精品在线开 放课程, 精品在线开放课程具有更高的平均选课人数, 其中, 工学类的精品在线开放课程的 选择人数达到了 6000 多人, 医学类的平均选课人数也超过了 5000 , 文科类的平均选课人数达 到 4000 人左右。

综上所述，河南省的在线开放课程建设主要特点及问题有: 
(1) 在线开放课程分布不均。河南省内排名前十名高校的在线开放课程建设指标为 101 门, 占比 $33.7 \%$, 而其中入选一流大学建设高校占比为 $39.6 \%$ 。这说明一流大学建设高校在开 放课程建设方面的人力、财力、物力都具有十分明显的优势。值得注意的是, 到目前为止, 河南省入选国家精品在线开放课程的门数不足 $10 门$, 其中包括了3所高职高专院校所建课程。 这一方面说明了河南省内众多高校在建设国家精品在线开放课程方面存在不少问题，另一方 面却也说明了省内高职高专院校通过全力打造课程能够很好的保证课程质量, 改善教学。

（2）学科门类覆盖范围广，学科间发展并不平衡，尤其是冷门学科的在线开放课程建设 严重落后。并且专业课程的在线开放课程建设热情高, 通识课与公选课建设占比少。一方面, 专业课程的内容集中, 专业知识覆盖范围窄, 建课相对容易, 专业教师参与相关课程教学多 年, 心得体会深, 对教学过程中的痛点、难点把握准确, 授课容易得到学生的认可与接受, 因此, 教师对课程的网络化、信息化响应积极。另一方面, 大部分高校为非综合性高校, 学 科门类不齐全, 加上又是冷门学科, 课程受众面窄, 教师对课程的在线转化意愿较低, 不愿 意花时间进行在线教学。

(3) 选课人数方面: 工、文、医学类学科选课人数多, 理、农学科选课人数偏少; 入选 国家精品课程的选课人数远大于非精品课程的选课人数。这一方面, 得益于精品资源课程资 源自身质量较好, 能够吸引大批学习者; 另一方面, 入选国家精品在线开放课程能够极大提 升课程影响力, 从而吸引更广范围、更多类型的学习者选课学习。然而, 由于国家精品在线 开放课程评选要求、数量的限制, 绝大部分具有特色的在线开放课程很难入选或者需要长时 间的积累与不断优化。因此, 有必要提供新的在线开放课程推广方法, 以提高选课人数, 扩 大课程影响力。

(4) 大数据分析技术在教学辅助过程中作用缺失。中国大学MOOC作为主流的在线开放 课程平台并且作为河南省所有在线开放课程上线运行平台, 具有强大的大数据分析潜力, 然 而, 从目前来看, 各课程的授课团队没有充分利用该平台的数据分析能力辅助教学过程。并 且在授课过程中交互不足, 特别是课堂讨论区中, 交互数明显偏低 ${ }^{[8]}$, 对学生的学习过程的管 理与监测, 对落后学生的督促, 对教学过程中的问题反馈等信息均没有充分利用起来, 无法 搭建与学生的互动通道, 导致教学效果不佳, 教学互动缺失等问题。

\section{4. 优化建议}

本文紧密结合当前互联网发展的新趋势，围绕教育信息化改革、教育资源共享，智慧教 育等在线开放课程发展目标，针对河南省在线开放课程建设存在的问题，分别从课程资源建 设、推广、应用等方面提出了建议与对策。

1、促进在线开放课程建设均衡发展。鼓励各高校积极投入，提高在线开放课程建设力度。 与此同时，在国家精品在线开放课程评价标准的基础上，建立在线开放课程资源建设与质量 保障标准, 用于指导课程建设与评价。除此之外, 考虑到各类高校在学科优势、师资力量、 资金供给、社会影响力等方面存在明显差异，各高校在努力发展在线开放教育的同时，必须 以自身办学特色、学科优势和教学质量为目标, 结合当前教育技术发展新趋势, 设计在线开 放课程建设路径, 保障有限资源利用的最大化和效率最优化。对于在线开放课程建设数量较 多但入选国家精品在线开放课程数量偏少的高校, 应具体分析其存在的问题, 改善其课程质 量, 增加入选数量, 提升高校教学质量。建设数量少, 但能够坚持质量优先, 从而入选国家 精品在线开放课程的特色院校, 应继续强化其课程质量, 推广其建设经验, 并且增加其课程 建设数量, 在保证质量的同时, 让其建设数量也能够得以发展, 以增加入选数量。总而言之, 各高校应进一步转变教学理念, 坚持自身办学特色, 以突出优势学科和改善教学质量为最终 导向，并确保该导向始终贯穿整个在线开放课程建设过程。除此之外，在政府部门主导下， 学校应配套相关学分、课时等政策, 积极参与在线开放课程建设过程, 引进校外优质资源以 弥补存在的学科劣势, 使得各学科能够得以均衡发展。 
2、充分利用图书馆的信息中转优势，促进在线开放教育资源质量优化与共享，扩大优质 在线开放课程覆盖面与影响力, 改善课程学习人数少的问题。在线开放课程的开放性决定了 课程资源建设的首要目标是最大限度范围的共享, 对于高校而言, 图书馆是信息中转站, 具 有信息资源丰富, 更新速度快, 覆盖范围广等特点。早在MOOC发展伊始, 国外高校图书馆 就深度介入了在线开放课程资源的管理、维护，以及推广等环节，一方面，高校可充分利用 图书馆的信息优势, 加大对在线开放课程的推广应用, 提高学校知名度, 并向教师提供资源 版权、版权许可, 版权结算等方面的指导; 另一方面, 在线开放课程资源的广泛应用与共享, 可提高一线教师的成就感, 充分调用一线教师利用开放课程资源开展创新教学的动力和潜能。 最后, 在课程资源建设过程中, 图书馆可提供包括图像、文本、音频，视频等各种版权或无 版权资源, 有助于提高课程资源质量与建设进度。在课程资源发布后, 图书馆可作为课程的 一个反馈窗口, 收集学习者的学习反馈信息, 并根据反馈, 及时提供在线开放课程需要的各 类参考资料。

3、利用统一平台优势, 夯实大数据基础, 建立关联模型, 优化教学管理和决策。在线开 放课程区别于传统教学模式的重要特征是学习者的学习行为数据被精确而详尽地记录下来, 并且具有数据量大、数据类型多样、数据准确等特征。通过对学习行为大数据的充分利用, 可获得对教育的新认知, 创造新的价值, 改变教育部门、教师, 学生的关系。在大数据的支 撑下, 可以实现教育资源的精准化建设、适应性的资源推送以及资源的智能分类与动态聚合, 有助于数字教育资源生态的持续进化。对于学习者来说, 通过在线课程学习平台的行为记录, 借助大数据分析技术, 可准确构建适用于自身学习特征的个性化学习模型, 实施个性化教育, 真正实现“因材施教”和“因人施教”。学习者是课程教学过程中的主体，在分析其学习行为的 基础上, 为了更好地服务教学目标, 可在教学过程中及时调整或变革教学内容、教学方法、 教学手段和教学内容组织方式等。在教学过程中, 既不放弃传统教学模式的优点, 同时结合 在线教育模式的长处, 改变传统单调的教学模式。

4、强化教师信息化应用水平, 改善信息化教学理念与意识, 提高师生信息素养。首先, 建立在线开放课程资源建设规范与流程，针对不同学科不同类型的课程给课程分门别类的制 定课程资源制作要求、制作模板、课程视频设计指导, 以及课程完整程度检查标准等; 其次, 构筑线上线下师生、生生间互动交流通道, 提高互动交流效率。虽然MOOC在规模, 开放性, 资源获取便利性方面具有先天优势, 然而, MOOC在知识传递成效和持续性方面的问题一直 得不到有效解决 ${ }^{[9-10]}$ 。针对该问题，可建立由主讲教师、助教、系统维护等不同层次水平的人 员组成的多层次互动交流通道。第一, 通过多层次交流, 可充分利用各层次水平, 不同功能 的人员的能力，及时解决各种问题；第二，对问题的及时解答，可极大地提高学习者交流积 极性, 让其能更好、更深入的参与日常教学过程, 并形成正反馈, 为整个交流互动系统带来 积极正面的影响，从而保证互动达到预期效果。

\section{致谢}

本文为河南省高等学校重点科研项目计划“MOOC环境下高校资源整合与共享的实现路 径研究” (项目编号：17B630004) 及河南省教育科学”十二五“规划2015年度课题“融合“CDIO” 理念的翻转课堂教学模式研究（项目编号：[2015]-JKGHYB-0630）的阶段性成果之一。

\section{References}

[1] Du Wen-chao, He Qiu-lin, Jiang Li-jun, The Pioneer of Sharing Course Resources in the world Analysis of MIT OCW Project, MODERN EDUCATIONAL TECHNOLOGY, 21(4), pp. 14-18, 2011. 
[2] Hylén J. Open educational resources: Opportunities and challenges[J]. Annals of Thoracic Surgery, vol.80, pp.969-975, 2011.

[3] Zang Quan-guo, An Ming. Practice and Reflection on Library 2.0 in Libraries at Home and Abroad[J]. Library and Information Service, vol.57, pp.51-56, 2013.

[4] Cheng Lin. Comparative Analysis of the mainstream MOOC platform[D].Nanjing University of Posts and Telecommunication, 2015.

[5] Qian Lu. A Survey of MOOC status[D]. Northeast Normal Univesity, 2016.

[6] Wu Jin-hui. Comparative Analysis of China's Main MOOCs Platform[J]. University library work, vol.1, pp.51-56, 2015.

[7] Li Xiu-li. Investigation and Analysis of College Mood Construction and Course Utilization in China-Taking the Four Platforms of Chinese University MOOC as an Example[J]. Researches in Library Science, vol.10, pp. 52-57, 2017.

[8] Fang Xu. Empirical Analysis of Affirmation of National Boutique Online Open Courses[J]. China Higher Education Research, vol.7, pp.98-103, 2018.

[9] Jia Ji-you, Liao Jing-ming, Wang Qiong. Big Data Analysis of MOOC Learning Behavior and Effect: A Case Study of 6 MOOCs in Peking University[J]. Indusrty and Information Technology Education, vol.9, pp.23-29, 2014.

[10] Wang Ji-de, Liu Ya-tong. Problem Analysis and Promotion Strategy Research on the Effect of College Students' Interdisciplinary MOOC Courses_-Taking the MOOC Platform of Chinese University as an Example[J]. Indusrty and Information Technology Education, vol.1, pp.5-11, 2017. 